\title{
Wohlfahrtskapitalistische Regime und Handlungsfähigkeit erwerbsloser junger Menschen in Spanien und Deutschland
}

\author{
Christoph Gille • Ute Klammer
}

Online publiziert: 8. Juni 2017

(C) Der/die Autor(en) 2017. Dieser Artikel ist eine Open-Access-Publikation.

Zusammenfassung Anhand der Möglichkeiten zur Herstellung von Handlungsfähigkeit junger Erwerbsloser in den wohlfahrtskapitalistischen Regimen von Deutschland und Spanien geht der Artikel der Frage nach, wie und mit welchen Ergebnissen sozialpolitische Regimeforschung und akteur*innenbasierte Forschungsansätze kombiniert werden können. Dabei werden zunächst zentrale Aspekte der Arbeitsmarktentwicklung und institutionellen Rahmenbedingungen beider Regime verglichen. Anschließend werden Ergebnisse einer qualitativen Untersuchung junger Erwerbsloser in beiden Ländern anhand von ausgewählten Bewältigungsweisen vorgestellt, bevor Charakteristika der Regime aus akteur*innenbasierter Perspektive erörtert und Schlussfolgerungen gezogen werden.

Schlüsselwörter Wohlfahrtskapitalistische Regime - Akteur*innenforschung • Jugendarbeitslosigkeit · Spanien · Deutschland

\section{Welfare capitalist regimes and agency of young unemployed in Spain and Germany}

Abstract This article looks at ways of how unemployed young adults gain agency in the welfare capitalist regimes of Spain and Germany and explores the question how findings of socio-political regime research can be combined with actor-based approaches, and what outcomes such a combination will yield. In a first step ma-

C. Gille, M.A. (₫)

Fakultät für Angewandte Sozialwissenschaften, Technische Hochschule Köln,

Ubierring 48, 50678 Köln, Deutschland

E-Mail: christoph.gille@th-koeln.de

Prof. Dr. U. Klammer

Institut für Arbeit und Qualifikation (IAQ), Forsthausweg 2, 47057 Duisburg, Deutschland

E-Mail: ute.klammer@uni-due.de 
jor aspects of job market development and the institutional framework of the two regimes are compared. Then we present the findings of a qualitative study of the courses of action young unemployed people can take based on selected modes of agency and move on to explain the characteristics of the welfare capitalist regimes from an actor-based perspective. Finally, conclusions resulting from this analysis will be drawn.

Keywords Welfare capitalist regimes · Actor-based research · Youth unemployment $\cdot$ Spain · Germany

\section{Regime und Akteur*innen}

In der vergleichenden Wohlfahrtsstaatsforschung hat in den vergangenen Jahrzehnten - anschließend an und in kritischer Auseinandersetzung mit Esping-Andersens „The Three Worlds of Welfare Capitalism“ (1990) - der Fokus zahlreicher Arbeiten auf der Bestimmung und Abgrenzung unterschiedlicher „Regime“ wohlfahrtsstaatlicher Regulierung und Leistungsgewährung in Europa gelegen. Im Zentrum steht dabei eine Betrachtung der institutionellen Strukturen und vorgesehenen generalisierenden Leistungen für typische Notlagen aus der Makro-Perspektive. In späteren Arbeiten wird der Blick bereits von einer funktionalistisch geprägten Konzentration auf sozialpolitische Institutionen auf die Kräftekoalitionen und Praktiken der Individuen geweitet, die zu ,relativ stabilen, politisch institutionalisierten Regulierungsarrangements" führen (Lessenich und Ostner 1998, S. 12). In diesem Sinn ist die Regimeforschung auch in der Wissenschaft der Sozialen Arbeit aufgegriffen und im Wesentlichen von Andreas Walther (2011, 2013) fruchtbar gemacht worden, der in seinem Konzept der Lebenslaufregime das Verhältnis zwischen Individuum und Gesellschaft insbesondere an den Übergängen im Lebenslauf in den Fokus rückt.

Der Ansatz der Regimeforschung bietet vor allem zwei Vorteile: Zum einen werden verschiedene Sphären der Wohlfahrtsproduktion einbezogen, die klassischerweise als Trias Familie/Markt/Staat bezeichnet werden. Damit geraten auch Elemente der Wohlfahrtsproduktion in den Blick, die nicht direkt oder nicht umfassend sozialpolitisch geregelt werden. Zum anderen werden verschiedene Ebenen wohlfahrtskapitalistischer Arrangements berücksichtigt, Walther (2013) unterscheidet hier zwischen der sozio-ökonomischen, institutionellen, kulturellen und individuellen Dimension. Mit Rückgriff auf Michel Foucault seien Regime als „Konfigurationen der Regulierung sozialer Beziehungen“ zu verstehen, die sowohl Prinzipien, wie Normen, Regeln und Institutionen umfassen (Walther 2011, S. 84). Insbesondere durch den Einbezug einer Ebene, in der die für die Einzelnen geltende symbolische Ordnung expliziert wird, geraten die Akteur*innen als Co-Produzent*innen der sozialen Ordnung mit in den Blick.

Forschung in der Wissenschaft der Sozialen Arbeit hat in den vergangenen Jahren mehr und mehr akteur*innenbezogene Perspektiven entwickelt und genutzt (für eine Übersicht: Graßhoff 2013). Ansätzen wie denen der Adressat*innen-, der Nutzer*innen- und der Nutzungsforschung ist gemeinsam, dass sie sich von den eindeutigen, institutionell vorgegebenen Logiken lösen und - auch als Reaktion auf eine 
einseitige politische Fokussierung auf neoliberale Wirksamkeit (vgl. Herzog 2015) - die Alltagspraktiken der Akteur*innen in den Blick nehmen. Einen Anschluss an die sozialpolitische Literatur bieten dabei die Arbeiten von Hans Steinert und Arno Pilgram (2001), die Wohlfahrtsproduktion „,von unten“ erforschen und damit die Relevanzsetzungen der Akteur*innen zum Ausgangspunkt nehmen. Gerade vor dem Hintergrund des transformierten Sozialstaats, in dem Sozialpolitik re-moralisiert und Selbstführung zum Prinzip wird (Lessenich 2008), stellt sich die Frage, wie auf die neo-sozialen Anrufungen reagiert und gesellschaftliche Teilhabe durch die vom Ausschluss Betroffenen hergestellt wird.

Auch in der neueren Erwerbslosenforschung liegt eine Reihe von Untersuchungen vor, in denen die Subjekte zum Ausgangspunkt gesellschaftlicher Analysen werden. Dazu können unter anderem die interaktionistischen Untersuchungen der Prozesse zwischen MitarbeiterInnen der Arbeitsverwaltung und Arbeitssuchenden von Wolfgang Ludwig-Mayerhofer et al. (2009) oder Kolbe (2013) zählen, die subjektorientierten Arbeiten zum Übergangsystem von Barbara Stauber et al. (2007) oder beispielsweise Enzo Weber (2014), die Nutzungsforschung zu arbeitsmarktpolitischen Maßnahmen von Anne van Rießen (2016, subjektorientiert hier Weber 2014) und auch die machtkritischen Erkundungen der Subjektpositionen, wie sie Dennis Eversberg (2014) der Erkundung der „Individualitätsklassen“ Erwerbsloser zugrunde legt. Zwar unterscheiden sich die genannten Arbeiten im Einzelfall deutlich in ihrem theoretischen und methodologischen Verständnis, gemeinsam ist ihnen jedoch eine Erkundung des Sozialen aus der Position der Subjekte heraus.

Die hier vertretene Position basiert auf einem relationalen Verständnis von Handlungsfähigkeit, das von einem grundsätzlich zur Selbstbestimmung fähigen Subjekt ausgeht und gleichzeitig die institutionelle und symbolische Hervorbringung und die damit verbundenen Ermöglichungen und Blockierungen des Alltagshandeln freilegen will (vgl. Raitelhuber 2013, Bareis 2012). Agency wird als ,graduierte Qualität“ verstanden und die Frage gestellt, ,,welches Ausmaß von eigensinniger Handlungsfähigkeit für die jeweilige soziale Konstellation kennzeichnend ist" (Scherr 2012, S. 113). In diesem Sinn stellen wohlfahrtskapitalistische Regime Kontexte und Ausgangspunkte der Subjekte dar, die das Handeln zwar nicht determinieren, aber einen Rahmen mit limitierenden und ermächtigenden Qualitäten aufweisen, in denen Handlungsfähigkeit hergestellt wird.

Vor dem Hintergrund dieser Überlegungen stellt sich die Frage, ob eine Verknüpfung der vorherrschenden Analyseansätze aus Sozialpolitik- und Sozialarbeitsforschung einen Mehrwert für beide Disziplinen bietet, Verkürzungen auf generalisierende oder individualisierende Perspektiven vermeidet und die Verwiesenheit beider Felder aufeinander sichtbar machen kann (vgl. Olk 2008). Dieser Zusammenführung der im Allgemeinen eher strukturalistisch angelegten Regimeforschung und der handlungstheoretisch ausgerichteten Akteur*innenforschung ist der vorliegende Beitrag gewidmet. Er geht auf der Basis einer empirischen Studie am Beispiel der Handlungsräume junger erwerbsloser Menschen im mediterranen Wohlfahrtsstaat Spanien und dem konservativen Wohlfahrtsstaat Deutschland der Frage nach, wie beide Perspektiven mit Hilfe einer akteur*innenbezogenen Regimeforschung füreinander fruchtbar gemacht werden können. Im Folgenden wird zunächst eine kontextualisierende kurze Übersicht über relevante strukturell-institutionelle Merk- 
male der beiden Länder gegeben. Im Anschluss werden exemplarisch Ergebnisse einer Studie über junge Erwerbslose in Spanien und Deutschland vorgestellt ${ }^{1}$ und Schlussfolgerungen gezogen.

\section{Strukturell-institutionelle Ausgangslage in Spanien und Deutschland}

Mit Deutschland und Spanien stehen zwei wohlfahrtskapitalistische Regime für junge Erwerbslose im Blickpunkt, deren strukturelle und institutionelle Merkmale sich deutlich voneinander unterscheiden. Betrachtet man vorliegende Systematisierungen der wohlfahrtsstaatlichen Arrangements für junge Erwachsene (Chevalier 2016; Walther 2011; Serrano Pascual 2007), konstatieren sie übereinstimmend eine geringe Ausstattung junger Menschen mit Ressourcen im spanischen Regime. Maria Amparo Serrano Pascual (2007) spricht vom „fragmented provision regime“, Andreas Walther (2011) vom ,unterinstitutionalisierten Übergangsregime“, für Tom Chevalier (2016) ist es durch ,,denied youth welfare citizenship“ charakterisiert. Für das deutsche Regime werden dagegen eine größere Ausstattung mit Ressourcen und ein stark beaufsichtigender Charakter diagnostiziert. Walther (2011) ordnet es als „,erwerbsarbeitszentriertes Übergangsregime“ ein, nach Chevalier (2016) zeichnet es sich durch ,monitored youth welfare citizenship“ aus. Im folgenden Abschnitt sollen anhand sozio-ökonomischer und institutioneller Aspekte ausgewählte Charakteristika verdeutlicht werden, die für junge Erwerbslose relevant sind.

2007 und 2013, im Abstand von nur sieben Jahren, erlebt Spanien den ökonomisch erfolgreichsten und verheerendsten Moment seiner jüngeren Geschichte. Während im Jahresdurchschnitt 2007 20,6 Mio. Menschen einer Beschäftigung nachgehen, sinkt die Zahl der Erwerbstätigen bis auf 17,1 Mio. in 2013. Die Zahl der Arbeitslosen steigt von 2 auf 6 Mio. - die Erwerbslosenquote verdreifacht sich. Junge Menschen sind von der Beschäftigungskrise am meisten betroffen, seit 2012 liegt ihre Arbeitslosenquote bei etwa 50\%. Zwischen 2008 und 2012 verlieren 1,9 Mio. junge Menschen zwischen 16 und 29 Jahren ihren Job, $67 \%$ aller Beschäftigungen gehen bei den Jungen verloren. Die deutlich sinkende Quote der erwerbsaktiven Unter-25-Jährigen weist darauf, dass immer mehr junge Menschen ihre Ausbildungszeiten verlängern. Stehen in 2008 noch $48 \%$ der Altersgruppe dem Arbeitsmarkt zur Verfügung, sind es in 2015 nur noch $35 \%$. Faktisch unterzeichnen damit die Arbeitslosenzahlen noch das tatsächliche Ausmaß der Jugenderwerbslosigkeit.

Blüte und Zusammenbruch des spanischen Arbeitsmarktes sind eng an die Dynamiken des europäischen Institutionengefüges und der Finanzmärkte gebunden. Mit der Aussicht und schließlich dem tatsächlichen Eintritt in die Eurozone 1999 setzt ein enormer Bauboom ein. Nicht nur für Spanier, auch für internationale Anleger erscheint es ohne Devaluationsgefahr sicher, in den spanischen Markt zu investieren. Zwischen 1997 und 2007 wächst der Wohnungsbestand um 7 Mio. Einheiten und es werden 1,6 Mio. Arbeitsplätze im Baubereich geschaffen (Lindner 2013; López und Rodriguez 2012). Doch mit dem Bankrott von Lehman Brothers und der darauf

\footnotetext{
1 Die empirischen Ergebnisse basieren auf dem laufenden Promotionsprojekt von Christoph Gille im Rahmen des Promotionskollegs „Leben im transformierten Sozialstaat“.
} 
Tab. 1 Daten zur Arbeitsmarktentwicklung in Spanien und Deutschland (2007-2015, in \%) (Eurostat, Arbeitslosenquoten nach ILO Definition)

\begin{tabular}{|c|c|c|c|c|c|c|c|c|c|}
\hline & 2007 & 2008 & 2009 & 2010 & 2011 & 2012 & 2013 & 2014 & 2015 \\
\hline \multicolumn{10}{|l|}{$\overline{\text { Spanien }}$} \\
\hline $\begin{array}{l}\text { Erwerbstätige } \\
\text { insgesamt } \\
\text { (in Mio. Perso- } \\
\text { nen) }\end{array}$ & 20,6 & 20,2 & 19,1 & 18,7 & 18,4 & 17,6 & 17,1 & 17,3 & 17,9 \\
\hline $\begin{array}{l}\text { Arbeitslosenquote } \\
\text { insgesamt }\end{array}$ & 8,3 & 11,3 & 18,0 & 20,1 & 21,7 & 24,8 & 26,1 & 24,5 & 22,1 \\
\hline $\begin{array}{l}\text { Arbeitslosenquote } \\
\text { U25 }\end{array}$ & 18,1 & 24,5 & 37,8 & 41,6 & 46,4 & 52,9 & 55,5 & 53,2 & 48,3 \\
\hline $\begin{array}{l}\text { Anteil der Ar- } \\
\text { beitslosen an } \\
\text { allen Personen } \\
\text { unter } 25\end{array}$ & 8,7 & 11,7 & 17,0 & 17,7 & 18,9 & 20,6 & 21,0 & 19,0 & 16,8 \\
\hline $\begin{array}{l}\text { Aktive Bevölke- } \\
\text { rung U25 }\end{array}$ & 47,9 & 47,7 & 45,0 & 42,7 & 40,9 & 39,0 & 37,8 & 35,7 & 34,7 \\
\hline \multicolumn{10}{|l|}{ Deutschland } \\
\hline $\begin{array}{l}\text { Erwerbstätige } \\
\text { insgesamt } \\
\text { (in Mio. Perso- } \\
\text { nen) }\end{array}$ & 37,4 & 37,9 & 37,8 & 37,3 & 38,1 & 38,3 & 38,6 & 38,9 & 39,2 \\
\hline $\begin{array}{l}\text { Arbeitslosenquote } \\
\text { insgesamt }\end{array}$ & 8,7 & 7,5 & 7,8 & 7,1 & 5,9 & 5,5 & 5,3 & 5,0 & 4,6 \\
\hline $\begin{array}{l}\text { Arbeitslosenquote } \\
\text { U25 }\end{array}$ & 11,8 & 10,4 & 11,1 & 9,8 & 8,5 & 8,0 & 7,8 & 7,7 & 7,2 \\
\hline $\begin{array}{l}\text { Anteil der Ar- } \\
\text { beitslosen an } \\
\text { allen Personen } \\
\text { unter } 25\end{array}$ & 6,1 & 5,5 & 5,8 & 5,1 & 4,5 & 4,1 & 4,0 & 3,9 & 3,5 \\
\hline $\begin{array}{l}\text { Aktive Bevölke- } \\
\text { rung U25 }\end{array}$ & 51,5 & 52,2 & 51,8 & 51,3 & 52,4 & 50,7 & 50,9 & 49,9 & 48,5 \\
\hline
\end{tabular}

folgenden Finanz- und Wirtschaftskrise platzt die Investitionsblase. Die spanische Bauwirtschaft verliert ab 2008 etwa 1,7 Mio. Arbeitsplätze (60\% des Sektors) und zeichnet damit für die Hälfte der zerstörten Arbeitsplätze in der Krise verantwortlich, erst mit weitem Abstand folgen Industrie und Dienstleistungen (vgl. Banyuls und Recio 2012, siehe Tab. 1).

In Deutschland dagegen verringert sich sowohl das Wirtschaftswachstum als auch die Beschäftigung in Folge der Finanzmarktkrise nur kurzzeitig und kehrt schon bald wieder auf den Wachstumspfad zurück. In 2015 erreicht die Beschäftigung mit fast 43 Mio. Erwerbstätigen einen Höchststand. Die Arbeitslosigkeit fällt mit 6,7\% auf ein Niveau, das im wiedervereinigten Deutschland noch nie erreicht wurde (Institut für Arbeitsmarkt und Berufsforschung [IAB] 2016). Hinter den Zahlen liegt zum einen der Erfolg des deutschen Produktionsmodells, das mit seinem auf Export ausgerichteten, hohen Industrieanteil durch die niedrigen Wechselkurse von der Krise sogar profitiert (vgl. Lehndorff 2014). Zum anderen ist die niedrige Arbeitslosenquote dem umfangreichen Umbau des Arbeitsmarktes geschuldet. Während das 
Arbeitsvolumen erst in den letzten Jahren das Niveau der beginnenden 1990er Jahre wieder erreicht, verdoppelt sich die Teilzeitquote in den vergangenen 20 Jahren von $19 \%$ auf $38 \%$ (E. Weber 2014). Gleichzeitig vergrößert sich in den 2000ern das Segment prekärer Beschäftigung deutlich. Leiharbeit breitet sich massiv aus und es entsteht ein eigenes Arbeitsmarktsegment für geringfügige Beschäftigung. Klaus Dörre et al. (2013) kennzeichnen Deutschland deswegen als ,,prekäre Vollerwerbsgesellschaft".

Wie in allen Industrieländern sind auch in Deutschland junge Erwachsene häufiger von Erwerbslosigkeit betroffen. Mit 7,2\% in 2015 liegt die Arbeitslosenquote der U25-Jährigen in Deutschland jedoch so niedrig wie in keinem anderen Land der EU. Dennoch lohnt sich eine genauere Betrachtung. Der häufig ins Feld geführte Vorbildcharakter des dualen Ausbildungssystems verdeckt die massive Verschiebung hin zu den Hochschulausbildungen und in den Übergangssektor (vgl. Baethge 2014). Sowohl das Angebot an Ausbildungsplätzen als auch die Zahl der Bewerber*innen ging in den letzten beiden Jahrzehnten deutlich zurück und auch in 2015 konnten 80.000 junge Erwachsene nicht in eine Ausbildungsstelle vermittelt werden. Zu ihnen kommen 90.000 Bewerber*innen, deren Verbleib unbekannt blieb und diejenigen, die als ,nicht ausbildungsreif“ eingestuft wurden. 270.000 junge Menschen begannen eine Maßnahme im Übergangssektor, Kursen, die in der Regel nicht mit einem formalen Bildungsabschluss verbunden sind. Junge Menschen in Maßnahmen fließen ebenfalls nicht in die offizielle Erwerbslosenstatistik ein, damit wird das Ausmaß der Beschäftigungslosigkeit von jungen Menschen in Deutschland aus mehreren Gründen unterzeichnet. Auf der anderen Seite zeigen 40.000 unbesetzte Ausbildungsplätze das zunehmende Passungsproblem am deutschen Ausbildungsmarkt, das auch auf hohe regionale Unterschiede am Arbeitsmarkt verweist (vgl. Bundesministerium für Bildung und Forschung (BMBF) 2016; Bundesagentur für Arbeit (BA) 2016). Zudem stellt sich der Arbeits- und Ausbildungsmarkt für junge Erwachsene als hochgradig segmentiert dar. Die hohen Arbeitslosenzahlen unter jungen Erwachsenen mit niedrigen formalen Bildungsabschlüssen verweisen auf die mangelnde Integrationskraft von sowohl Ausbildungs- wie Übergangssystem (vgl. Reißig 2016). Während in Spanien 2015 die Arbeitslosenquote 25- bis 29-Jähriger mit niedrigen Bildungsabschlüssen mit knapp $40 \%$ etwa doppelt so hoch liegt wie jene der Jugendlichen mit hohen Abschlüssen, liegt sie bei ihren deutschen Altersgenossen bei 19\% und damit fünfmal höher als die Quote der Hochqualifizierten (Eurostat 2015).

In Spanien steht dem krisenanfälligen Produktionsmodell eine Struktur wohlfahrtsstaatlicher Absicherung gegenüber, die im Bereich der Arbeitslosen- und der Grundsicherung fragmentarisch bleibt. Die Arbeitslosenversicherung gewährt eine Leistungsauszahlung für solche Erwerbslose, die in den vergangenen 6 Jahren mindestens 360 Tage sozialversichert tätig waren. Sie können Ansprüche auf Arbeitslosengeld geltend machen, das bis zu zwei Jahre ausgezahlt und in Abhängigkeit vom früheren Verdienst und der Zahl der Haushaltsangehörigen zwischen 500 und $1400 €$ liegt. Nach sieben Jahren Erwerbsarbeitskrise zählt kaum noch ein junger Erwachsener zum Kreis der Berechtigten. Als Reaktion auf die hohe Erwerbslosigkeit hat der Föderalstaat Fürsorgeprogramme entwickelt, die wie die Renta Activa de Inserción zeitlich begrenzt sind und deren monatliche Leistungen $500 €$ nicht 
überschreiten. Unter-25-Jährige sind von der Inanspruchnahme dieser Programme ausgeschlossen. Mit zunehmender Dauer der Beschäftigungskrise hat sich die Zahl der Leistungsberechtigten dieser Transferleistungen deutlich verringert, in 2015 erhalten nur noch $55 \%$ der gemeldeten Arbeitslosen irgendeine Form zentralstaatlicher Unterstützung. Im gleichen Jahr machen Unter-25-Jährige nur noch $3 \%$ der Leistungsberechtigen aus und demonstrieren damit den geringen Grad der Absicherung junger Erwerbsloser durch den Zentralstaat (Servicio Público de Empleo Estatal [SEPE] 2015).

Diese zentralen Leistungen werden ergänzt durch Instrumente der Grundsicherung, die in den 1990er Jahren in allen autonomen Gemeinschaften eingeführt wurden (vgl. Alonso und Gozalo 2000). Die konkrete Ausgestaltung ist sehr unterschiedlich, so liegt z. B. das Leistungsniveau zwischen $44 \%$ und $100 \%$ des Minimumlohns. Trotz der Erwerbsarbeitskrise steigen Umfang und Leistungen der Grundsicherungssysteme nicht an, stattdessen führen Verschärfungen in den Bedürftigkeitsprüfungen zu deutlich höheren Ablehnungsquoten. In Katalonien etwa, wo die empirische Untersuchung durchgeführt wurde, erhöhte sich die Anzahl der Haushalte, in der kein Mitglied erwerbstätig ist, von $2 \%$ auf $13 \%$. Die Anzahl der Haushalte, die die dortige Grundsicherung (RMI) beziehen, liegt aber weiterhin deutlich unter $1 \%$ (vgl. Gutiérrez 2014). Auch von der Grundsicherung bleiben Unter-25-Jährige per Gesetz ausgeschlossen. Die katalanische Norm kennt wenige Ausnahmen von der Regel, zum Beispiel wenn junge Erwachsene eigene Kinder betreuen. Als Folge der rigiden Zugangsregeln sind im Jahr 2014 nur 2,9\% der RMI-Bezieher*innen jünger als 25 Jahre. Für alle autonomen Gemeinschaften fällt der Prozentsatz mit 3,4\% leicht höher aus (Ministerio de Sanidad, Servicios Sociales e Igualdad (MSSSI) 2015).

An der schwachen Ausstattung junger Erwachsener mit sozialen Rechten ändert auch die durch die Kommission und den Europäischen Rat in 2013 beschlossene, so genannte „Jugendgarantie“ nichts. 2,8 Mrd. Euro werden Spanien zur Umsetzung für den Zeitraum von 2014 bis 2020 aus dem Europäischen Haushalt bereitgestellt, wobei der größere Anteil aus dem Europäischen Sozialfonds zu diesem Zweck umgewidmet wird. Der Föderalstaat listet in seiner Jugendbeschäftigungsstrategie (EEEJ, Gobierno de España 2013a; 2013b) öffentlichkeitswirksam hundert Maßnahmen für junge Erwerbslose auf, darunter Abgabenerleichterungen, Förderungen von Selbstständigkeit und regional zu implementierende Qualifizierungsprogramme. In Katalonien konkretisiert sich die „Jugendgarantie“ im Plan Inserjoves. Konkret bauen etwa die beiden wichtigsten arbeitsmarktpolitischen Trainingsprogramme FEM und JOVES ihre Plätze von 3500 in 2013 auf etwa 5500 in 2015 aus. Angesichts von 115.000 bei der Arbeitsverwaltung gemeldeten erwerbslosen Unter 25-Jährigen in der autonomen Gemeinschaft wird die geringe Reichweite der Maßnahmen deutlich (vgl. Cabasés und Pardell 2014).

Im Unterschied zu Spanien hält Deutschland ein umfangreiches System sozialer Absicherung im Falle der Erwerbslosigkeit vor. Die Arbeitslosenversicherung sieht nach einer versicherten Beschäftigung von mindestens einem Jahr Lohnersatzleistung von bis zu einem Jahr in Höhe von 60 oder $67 \%$ des letzten Einkommens vor. Danach oder im Falle von fehlenden Anwartschaftszeiten erhalten Erwerbslose das steuerfinanzierte Arbeitslosengeld II, das einer alleinstehenden Person Leistungen in Höhe von $404 €$ plus Übernahme der Mietkosten auszahlt. Beide Systeme erreichen 
gemeinsam $89 \%$ der registrierten Arbeitslosen (BA 2016). In den 2000ern wurden die Leistungen für Erwerbslose starken Veränderungen unterworfen. Im Zuge der Hartz-Reformen materialisiert sich im Feld der Arbeitsmarktpolitik besonders deutlich der Pfadwechsel hin zur neo-sozialen Logik des so genannten ,aktivierenden“ Wohlfahrtsstaats, in dem die Pflicht zur Erlangung von Beschäftigungsfähigkeit im Zentrum steht (vgl. Mohr 2009). Besonders betroffen von den Veränderungen sind Erwerbslose Unter 25 Jahren. Zwar sehen die Regelungen weiterhin eine eigenständige Absicherung durch ALG II im Falle von Erwerbslosigkeit vor. Jedoch verfügen Unter 25-Jährige seit 2006 nur noch über einen elternunabhängigen Anspruch, wenn sie bereits vor Beginn der Arbeitslosigkeit einen eigenen Haushalt geführt haben oder sich die familiäre Situation als zerrüttet darstellt. Außerdem werden junge Erwerbslose deutlich häufiger zu den so genannten „Maßnahmen zur Aktivierung“ verpflichtet und ihre Sanktionierungsquote liegt etwa dreimal so hoch wie die der 25 bis 64-Jährigen (Achatz et al. 2012; Götz et al. 2010). Im Unterschied zu den Über 25-Jährigen sieht die gesetzliche Regelung für junge Erwerbslose die Möglichkeit einer hundertprozentigen Sanktionierung vor, sogar die Miete kann ihnen verweigert werden. Im Anschluss an die eingangs dargestellten Diagnosen treten mit den beschriebenen Veränderungen sowohl die umfangreiche Ausstattung mit Ressourcen als auch der paternalistische Charakter des deutschen Regimes zutage.

\section{Junge Erwerbslose in Spanien und Deutschland - ein qualitative Studie}

Vor dem Hintergrund dieser strukturellen und institutionellen Rahmenbedingungen stellt sich die Frage, welche Möglichkeiten zur Herstellung von Handlungsfähigkeit sich für junge Erwerbslose in den wohlfahrtskapitalistischen Arrangements in Deutschland und Spanien zeigen. Handlungsfähigkeit wird dabei unter Rückgriff auf die Theorie der Lebensbewältigung von Lothar Böhnisch als relationales Konzept verstanden (Böhnisch et al. 2009; Böhnisch und Schröer 2001). Die auf der Coping-Forschung beruhende Ausgangsannahme besagt, dass Menschen in allen Lebenssituationen Handlungsfähigkeit zu verwirklichen suchen, die auf das „Magnetfeld psychosozialen Strebens" - Selbstwert, Selbstwirksamkeit und Anerkennung - gerichtet sei (Böhnisch et al. 2009, S. 40). Dabei muss dieses Streben in Auseinandersetzung mit den gesellschaftlichen Bewältigungsaufforderungen und den zur Verfügung stehenden Ressourcen umgesetzt werden. Böhnisch entwickelt ein Modell dreier Zonen, die ineinandergreifen und weist damit auf die enge Eingebundenheit personaler Handlungsfähigkeit in spezifische Milieukontexte und sozialpolitische Strukturen hin. In der Verflechtung von Bewältigungslage und individuellem Handeln ist die Frage nach der Handlungsfähigkeit als relationales Konzept angelegt.

Die empirische Basis der Untersuchung bilden narrativ-episodische Interviews (vgl. Flick 2011, S. 238-247; Rosenthal et al. 2006) mit jungen Erwerbslosen zwischen 18 und 26 Jahren in Spanien und Deutschland. In die Auswertung sind 27 in Spanien und 25 in Deutschland geführte Interviews eingeflossen, die in der Provinz Barcelona und im Ruhrgebiet geführt wurden. Für das Sampling wurden drei soziodemographische Merkmale zur Kontrastierung ausgewählt: Geschlecht, Herkunft 
und Bildungsgrad, wobei die Aspekte Herkunft und Bildungsgrad an der Verteilung im jeweiligen Land orientiert sind. Im Schnitt waren die Befragten in Deutschland 23 und in Spanien 22 Jahre alt, die durchschnittliche Interviewdauer betrug 83 Minuten. Eine Hälfte der Interviews erfolgte mit jungen Menschen, die sich zum Zeitpunkt der Befragung in Programmen aktiver Arbeitsmarktpolitik befanden. Die andere Hälfte wurde mit jungen Erwachsenen außerhalb solcher Programme geführt, um sowohl Narrationen mit als auch ohne Einfluss der Institutionen analysieren zu können.

Die Auswertung erfolgte auf Basis der Grounded Theory (Strauss 1998; Strauss und Corbin 1994), wobei unter Rückgriff auf zwei Ideen bei Anselm L. Strauss (1998) sowie Gabriele Winkler und Nina Degele (2009) während der Codierung drei Ebenen unterschieden wurden: personale Bewältigung, symbolische Ordnung und institutionelle Strukturen. Der Ausgangspunkt der akteur*innenbasierten Erfassung der Regime liegt insbesondere in der Zusammenführung von symbolischer Ordnung und biografisch wirkenden, institutionellen Einflüssen, wie sie übergreifend in den Narrationen der jungen Erwerbslosen aus einem Land gefunden wurden. Die Bewältigungsweisen finden ihren Ursprung in der Auswertung der personalen Ebene und der Zusammenfassung der sich wiederholenden Elemente. Zugleich stellen die Ergebnisse immer ein Zusammenspiel aller drei Ebenen dar. So ist das Streben nach personaler Handlungsfähigkeit aufs engste mit verschiedenen symbolischen Ordnungen und institutionellen Einflüssen verflochten, die im individuellen Handeln erkennbar werden und im Folgenden auch an den zwei ausgewählten Beispielen erörtert werden. Die akteur*innenbasierten Regimecharakteristika hingegen sind Extrakte einer überindividuellen Orientierung, wie sie in den biografischen Narrationen der jungen Erwerbslosen jeweils eines Landes erkennbar werden. Sie sind das Ergebnis einer Gegenüberstellung der wesentlichen Merkmale beider Länder, in der insbesondere die Unterschiede der beiden Regime zur Sprache kommen.

\subsection{Bewältigungsweisen}

Sechs Bewältigungsweisen, denen eine zentrale biografische Bedeutung zukommt, wurden in den Interviews mit jungen Erwerbslosen in beiden Ländern identifiziert. Sie werden durch die Titel „Straße“, „Fürsorge“, „Pseudo-Arbeit“, „Befreiung“, „Erkenntnis“ und „Ausbildung“ markiert. Auf dieser Analyseebene unterscheiden sich die Ergebnisse in Spanien und Deutschland nicht. Vielmehr wird deutlich, dass sich der ,innere Kreis“ im psychodynamisch angetriebenen und sozial gerichteten personalen Bewältigungsverhalten (Böhnisch et al. 2009, S. 40) in zentralen Ausprägungsformen in beiden Ländern gleicht. Diese sechs Bewältigungsweisen sind nicht unmittelbar jeweils einer Person zuzuordnen, stattdessen können sie sich biografisch abwechseln oder werden ergänzend zueinander verfolgt. Alle Bewältigungsweisen zeichnen sich durch eine innere Ordnung aus - ein jeweils spezifisches Set zentraler Versprechen, Handlungsprinzipien und Limitationen, die voneinander unterschieden werden können. Diese überindividuelle innere Ordnung gibt den jungen Erwerbslosen einen gewissen Grad an Handlungsstabilität, die bei der Verwirklichung von Handlungsfähigkeit Orientierung gibt und zugleich ko-produziert wird. Am Beispiel zweier gegensätzlicher Bewältigungsweisen - „Straße“ und „Ausbildung“ soll in Auszügen und an wenigen Fallbeispielen erläutert werden, welche innere 
Ordnung jeweils gilt. Diese beiden Bewältigungsweisen wurden unter anderem ausgesucht, weil in ihnen die Bedeutung der sozialen Schichtung in beiden Regimen erkennbar wird. Während „Ausbildung“ nur solchen Befragten offen steht, die finanziell abgesichert sind, gibt die „Straße“ eine Möglichkeit eigenständiger materieller Existenzsicherung. Auf den Zusammenhang mit den wohlfahrtsstaatlichen Regimen werden wir noch zurückkommen.

\subsubsection{Straße}

Die Straße ist nicht nur häufiger Aufenthaltsort sondern auch Symbol einer Jugend, die ihr Leben da verbringt, wo andere auf dem Weg sind. Die Straße, eigentlich ein Ort, den man mit Ziel betritt, wird zu einem Ort des Verweilens. Ihre zeitliche Ordnung ist bestimmt durch das Fehlen des Morgens und des Wohin, stattdessen markiert durch eine Monotonie der Gegenwart: „okay, das war jeden Tag das gleiche, diese Monotonie, war .. war die gleiche Monotonie jeden Tag “ (Martin, 19, Spanien). Die zentralen Versprechen des Lebens auf der Straße sind zwei: Erstens die unmittelbare materielle Absicherung durch das Begehen delinquenter Handlungen, vor allem in Form von kleinkriminellem Diebstahl oder Dealen. Murad (21, Spanien) erklärt: „Früher hatte ich kein Geld, du gehst auf die Straße, verkaufst was, ich hab Marihuana, du vertickst .. sehr schlecht, weisst du?" Das zweite Versprechen ist das Erleben unmittelbarer Selbstwirksamkeit in den gleichen Momenten der Delinquenz, die Sicherheit, dass durch eigenes Handeln Einfluss auf die soziale Umgebung und auf das eigene Leben ausgeübt werden kann.

Diese Versprechen sind mit einer sozialen Ordnung innerhalb einer subkulturellen Gemeinschaft verwoben. Gerade weil man außerhalb der gesetzlichen Normen steht, werden Zugehörigkeit zum einen und unbedingte Loyalität zu den innerhalb der Gruppe geltenden Normen zum anderen zu den notwendig stabilisierenden Elementen der Bewältigungsweise. In den Gruppen werden Verhaltenscodices ausgebildet, die Handeln mehr oder weniger explizit festlegen. Normen verschwinden nicht, sie werden noch wichtiger in einer Umgebung, die eine Gegenordnung zu den gesellschaftlich vorherrschenden Regeln konstituiert. In regelmäßigen Ritualen vergewissern sich die Straßencliquen, wer zu der geltenden Ordnung dazu gehört. Sergej (23, Deutschland) berichtet: „Wir waren immer auf der Suche nach Geld und Feiern und keine Ahnung so. Das war einzigste bei uns. Dass wir so, immer so bis zum Abends alles haben, irgendwie auch Bude, irgendwas, Alkohol und und und und, alles [...] waren immer nur feiern, die ganzen vier Jahre, glaube ich. Die ganzen vier Jahre. Einfach weg. Einfach irgendwie so, so ein Leben, dass du nix musst. Du hast das nicht, du - ich musste mich bei keinem melden, bei meiner Mutter [...] als das angefangen mit Buden hat, also so Wohnungen. Dann ham wir schon Hallen gemietet. Und dann - Dann kamen schon vom Nachbarstädten, ja, so. Die ganzen Russen, dann waren wir ja schon locker 100, 200, ungefähr. Da waren richtig viele. Und dann, [lacht] das war heftig, halt." Dass Sergej hier zunächst formuliert, dass er „nix muss“, relativiert er sofort. Er muss nichts mehr gegenüber seiner Mutter. Aber er ist neue Verpflichtungen zu spezifischen Formen der Kooperation eingegangen, die sich unter anderem in der beständigen Rede vom ,wir" zeigen, dem kollektiven Akteur, durch den Handlungsmacht hergestellt wird. In allen Straßenordnungen 
gilt das Prinzip der gemeinsamen Haushaltung, Sergej spricht von den „Buden“, in denen gemeinschaftlich gewirtschaftet wird. Was einer erwirbt, erwirbt er für die Absicherung der Gruppe und stabilisiert so das Überleben der Gemeinschaft. Außerdem gelten Mechanismen verpflichtender Reziprozität, in der die Einzelnen sozial kontrollierte und von Machtverhältnissen strukturierte Beiträge zur Versorgung leisten müssen. Gemeinsame Haushaltung, verpflichtende Reziprozität und Delinquenz bilden die Basis der ökonomischen Absicherung.

„Heftig“, sagt Sergej am Schluss über seine Zeit in den Buden und am Fluss. Eine Formulierung, die darauf hinweist, dass die Bewältigungsweise „Straße“ auch Risiken birgt. In der Formulierung „Knast oder Tod“ (Ben, 20, Deutschland) sind die zentralen Bedrohungen auf den Punkt gebracht. Mit Knast ist die Gefahr des Statusverlustes und der sozialen Exklusion durch den Eingriff der staatlichen Ordnung benannt. Mit Tod die physische Gefahr, die z. B. aufgrund der prekären Versorgungslage oder durch die Konkurrenz mit anderen Kollektiven ein nicht unwahrscheinliches Risiko darstellt. Murad: „Also, ich war bei ihm zuhause, um was zu kaufen, Marihuana zu kaufen [...] ich nehm das Zeug und bring's zu mir und das war, ich weiss nicht, eine Scheisse, eine Scheeeisssee, das Zeug, aber wirklich eine Scheisse. Und ich sach, Leo, was ist das?' [...] Und er war 29, okay, riesengroß, , Wie, wie was ist das?' und der zieht mir eine drüber, hier. Und ich reg mich auf, geh weg, geh und kauf mir ein Pfefferspray und geh wieder zurück zu seiner Wohnung, der macht mir auf, ich sprüh ihn voll, ich zieh ihm eine drüber. "Murad ahndet den Regelverstoß und sorgt durch sein Handeln mit für die Verbindlichkeit der geltenden Verhaltenscodices. Nicht immer so drastisch wie in der Schilderung von Murad finden sich in den Interviews zahlreiche Sanktionierungen, die für die Einhaltung sozialer Normen sorgen, darunter Anerkennungsverluste oder Ausschlüsse aus den gemeinsamen Haushalten. Was sich vermeintlich als ein anomisches Leben gestaltet, in dem man ,nix muss “, erweist sich als eine hochgradig verregelte Bewältigungsweise mit klaren Versprechen, strikten Regeln und deutlichen Risiken.

\subsubsection{Ausbildung}

Der Straße soll hier eine Bewältigungsweise gegenüber gestellt werden, die auf einer dominanten gesellschaftlichen Zielsetzung beruht und damit als konforme soziale Bewältigungsweise gelten kann (vgl. Merton 2016 [1968]). In der Bewältigungsweise Ausbildung greifen junge Erwachsene das Versprechen des kapitalistischen Marktes auf, versuchen ihre Arbeitsmarktposition durch ein höheres Niveau formaler Bildung zu verbessern und so ihre Chancen auf Berufseinmündung zu erhöhen. Verde (23, Spanien), mit Bachelor in einem naturwissenschaftlichen Fach, beschreibt, wie sie sich dem gängigen Muster der Menschen ihres sozioökonomischen Milieus in der Erwerbsarbeitskrise entziehen wollte und direkt im Anschluss an den Bachelor auf die Suche nach einem Arbeitsplatz gegangen ist: ,Und klar, der Moment während des Studiums, ja? Vielleicht war ich, hatte ich viele Eigenschaften, vielleicht war ich ein bisschen rebellisch, ja? Gegen, also so gegen wie man das so macht, ja? Des Systems, ja? [...] Also in diesem Sinne, in diesem akademischen Sinn, dass ich gesagt habe, dass ich keinen Master machen werde, ja? Weil, also weil einfach alle einen Master gemacht haben und ich wollte keinen Master machen, weil ich 
gedacht hab, ich hab mich schon genug angestrengt, um Biochemie zu studieren und ich dachte, das würde schon genügen. " Durch das Scheitern bei der Suche nach einem Arbeitsplatz gibt Verde ihre „Rebellion“ auf. Die Arbeitslosigkeit bedroht ihre Anerkennungsperspektive und verstrickt sie in größere materielle Abhängigkeit. In der Folge passt sie sich an die Erwartungshaltung ihres Herkunftmileus an und wählt mit dem weiterführenden Studium die für ihr Milieu typische Bewältigungsweise.

Auch Caro (25, Deutschland) verfügt über hohe Bildungstitel, einen internationalen Bachelor und einen internationalen Master und ist seit über einem Jahr arbeitssuchend. Sie überlegt, ihre Position am Markt durch Praktika zu verbessern: „Weil es mir wirklich nicht mehr ums Land geht, sondern gerade nur noch um irgendeine Art von Arbeit in dem großen Ganzen. Genau. Was sich schon enorm weiter oder was sich schon enorm geöffnet hat. [...] Hauptsache, ich krieg die Erfahrung und es ging von, bis vor drei Monaten war es Hauptsache bezahlt. Jetzt geht es zu Hauptsache irgendwas. "Caro hat bereits Kompromisse bei der Suche gemacht und ihre Präferenzen „,enorm geöffnet“. Dennoch geht es ihr eben nicht um ,,irgendeine Art von Arbeit“, sondern um „,irgendeine Art von Arbeit in dem großen Ganzen“. Kompromisse kann sie nur insoweit gehen, als sie der gesellschaftlichen Position Genüge tun muss, in der sie sich befindet. In der Folge hält Caro nur bestimmte Optionen offen, andere werden verworfen. Mit der Bewältigungsweise „Ausbildung“ ist bei allen Befragten immer das Versprechen gesellschaftlichen Aufstiegs oder zumindest der Beibehaltung der gesellschaftlichen Position verbunden. Ein Versprechen, das je nach Herkunft auch zu einer habituellen Verpflichtung werden kann.

Eine Voraussetzung der Bewältigungsweise „Ausbildung“ liegt in der ökonomischen Möglichkeit, sie zu realisieren. Lebenshaltungskosten und Kosten der Ausbildungsgänge müssen getragen werden können. Die Bewältigungsweise steht also nur denjenigen jungen Erwachsenen zur Verfügung, die über solche ökonomische Absicherung verfügen. Für Caro und Verde ist das der Fall. Gleichzeitig wird bei ihnen deutlich, dass mit der Finanzierung auch eine Verpflichtung gegenüber den Eltern verbunden ist. Aufgrund der ökonomischen Abhängigkeit ergeben sich die Entscheidungen über den weiteren Ausbildungsweg im Zusammenspiel zwischen Erwartungen der Eltern und den eigenen Einschätzungen und Wünschen.

Die größte Gefahr der Bewältigungsweise „Ausbildung“ liegt in der Möglichkeit, dass der Markt sein Versprechen nicht einlöst. Wer länger erwerbslos ist, hat diese Erfahrung bereits gemacht. Bei Verde bleibt ein grundsätzliches Misstrauen in das Marktversprechen und mit ihm eine Skepsis gegenüber der Verfügbarkeit der Zukunft zurück: „Das, was du hörst, ist nicht das, was du erfahren hast. [...] Also mir gibt das kein, ich weiß nicht, letztlich hab ich kein Vertrauen. Auch wenn ich, also ich würde es versuchen, ich weiß nicht. Also jetzt im Moment glaub ich, dass ich es doch versuche. Aber ich habe nicht besonders viel Hoffnung, weißt du? " Die Situation massiver Arbeitslosigkeit, von der breite Bevölkerungsschichten in Spanien betroffen sind, generiert bei Verde einen anderen Zweifel mit anderen Auswirkungen auf die individuelle Bewältigung als bei Caro. Diese übereinstimmend gefundenen, länderspezifischen Formen der Rationalisierung des Arbeitsmarktes und der eigenen Verantwortung verweisen auf übergreifende Charakteristika der wohlfahrtskapitalistischen Regime, in denen die jungen Erwachsenen ihre Handlungsfähigkeit herstellen und bilden damit eine Brücke zur zweiten Ebene der Untersuchungsergebnisse. 


\subsection{Akteur*innenbasierter Regimevergleich}

Als - angesichts der unterschiedlichen Rahmenbedingungen zunächst überraschendes - Ergebnis der empirischen Analysen lässt sich festhalten, dass die übereinstimmenden Bewältigungsweisen zeigen, wie ähnlich junge Arbeitslose in Spanien und Deutschland Handlungsfähigkeit herstellen. In beiden wohlfahrtskapitalistischen Regimen sind bestimmte gesellschaftliche Positionen mit vergleichbaren Handlungsmöglichkeiten versehen. Auf einer zweiten Ebene sollen jetzt diese gesellschaftlichen Kontexte sichtbar gemacht werden, also der äußere Kreis der Bewältigungsaufforderungen, in denen Limitationen und Ermöglichungen der Regime wirken und reproduziert werden. Dafür erweisen sich unter anderem die Grenzen der jeweiligen Bewältigungsweisen als wichtige Indikatoren. Welche Formen der Handlungsfähigkeit dem einen oder dem anderen jungen Erwachsenen in Deutschland oder Spanien verwehrt sind, weist häufig auf charakteristische Elemente von Markt, Staat und Gemeinschaft. Anhand solcher Momente und auf der Basis der gesamten biografischen Narrationen und den darin rekonstruierten personalen, symbolischen und institutionellen Ordnungen bildet sich ein akteur*innenbezogenes Bild der beiden wohlfahrtskapitalistischen Regime heraus, wie es sich aus der Perspektive der Subjekte im Vergleich bestimmen lässt. Tab. 2 gibt einen Überblick über die so gewonnenen, wesentlichen Charakteristika. Jedes darin genannte Merkmal fasst eine große Anzahl übereinstimmender symbolischer Orientierungen und institutioneller Einflüsse zusammen, wie sie in den Narrationen zu finden sind. In jedes Charakteristikum fließen also sowohl im empirischen Material sichtbar werdende, biografisch wirkende Rationalisierungsweisen als auch institutionelle Formierungsweisen ein (vgl. Kessl 2013). Anhand der aufgeführten Fallbeispiele und der in ihnen sichtbar gemachten Bewältigungsweisen sollen im Folgenden einige Aspekte - Markt als Lotterie und fehlender Wohlfahrtsstaat in Spanien, sowie rigorose Subjektivierung und zuweisender Wohlfahrtsstaat in Deutschland - in Auszügen vorgestellt werden.

Tab. 2 Differierende Ausprägungen von Markt, Staat und Gemeinschaft im akteur*innenbasierten Regimevergleich

\begin{tabular}{lll}
\hline & Spanien & Deutschland \\
\hline Markt & Markt als Lotterie & Markt als Konkurrenz \\
& Patronage & \\
Staat & Fixierte Prekarität & Institutionalisierte Prekarität \\
& kollektive Erwerbslosigkeit & individualisierte Erwerbslosigkeit \\
& relativierte Subjektivierung & rigorose Subjektivierung \\
& Fehlender Wohlfahrtsstaat & Zuweisender Wohlfahrtsstaat \\
& Rechte verweigernder Staat & Rechte gewährender Staat \\
& & Rechte gefährdender Staat \\
Gemeinschaft & Gemeinschaft als Zwang & Gemeinschaft als Option \\
\hline
\end{tabular}




\subsubsection{Spanien}

Murad und Verde etablieren ihr jeweiliges Bewältigungsverhalten vor dem Hintergrund eines Marktes, der sein Versprechen auf ökonomische Integration nicht mehr einlöst. In der Folge wird die Einmündung in den Arbeitsmarkt zur Lotterie, zu einem Glücksspiel, bei dem die persönliche Eignung nur eine geringe Rolle spielt. Murad: „Neeein, da muss man gar nichts machen, das ist einfach das Glück, das man so hat, verstehst du? Vielleicht gibt es ja ne Menge Leute wie mich und die haben mehr Glück. Schau mal, ich hab viele Bewerbungen eingeworfen und mich hat niemand angerufen. Aber sicher hat irgendein anderer in meiner Situation eine Bewerbung eingeschmissen und ihn haben sie angerufen, weißt du? Du musst gar nichts machen, es ist, was weiß ich, es ist einfach, da muss einfach einer auf mich setzen, verstehst $d u$ ?" In den spanischen Interviews wimmelt es, wie im Ausschnitt von Murad, vor allem vom „echar" und ,tirar“, einem „Werfen" der Bewerbungen ohne konkreten Empfänger und ohne eine begründete Aussicht. Alleine die Hoffnung auf das Losglück bleibt die Verpflichtung der jungen Erwerbslosen; sie macht es möglich, Handlungsfähigkeit im Heute herzustellen (vgl. Bourdieu 2000 [1977]). Murad: ,Und weiter nichts, verstehst du? Es ist so ... keine Ahnung. Einfach auf das Glück warten! Klar! “

Der spanische Wohlfahrtsstaat verweigert den jungen Erwachsenen eine eigenständige Absicherung. Wenn die jungen Erwerbslosen dennoch mit sozialstaatlichen Instrumenten in Berührung kommen, können sie sie im Bereich des Arbeitsmarktes kaum als Institutionen lesen, die auf sozialen Rechten beruhen. Durch ihre Partikularität werden sie stattdessen von den Logiken der beiden wohlfahrtskapitalistischen Sphären Markt und Familie überlagert. So auch bei Murad. Zwar nahm er an einer Maßnahme aktiver Arbeitsmarktpolitik teil, liest sie aber wie die anderen Befragten zunächst vor allem als reguläre Erwerbsarbeit. Er „,bewirbt“ sich, wird „,angenommen“, erhält „Arbeit“, die er „,bezahlt“" bekommt, auch wenn es sich nur um $150 €$ für die sechs Monate des Programms handelt. So verwandelt sich die Maßnahme aktiver Arbeitsmarktpolitik in reguläre Arbeit, bei der man bei der Gemeinde angestellt ist und die demselben Prinzip des Marktes als Lotterie unterworfen ist. In diesem Fall hatte Murad „Glück“.

Zum anderen werden wohlfahrtsstaatliche Institutionen durch Logiken überformt, die der Sphäre der Freunde und Familie entstammen. Murad führt die MitarbeiterInnen der Arbeitsverwaltung direkt mit Namen ein: „Es gab einen Fabi, glaub ich, einen Typen der Fabián hieß, mit Brille, glaub ich [...]. Früher lief das gut hier, da bist du da hin gegangen, die haben viel gesucht. Jetzt auch, ja! Die Armen, die versuchen dir zu helfen, aber die Situation im Land ist jetzt so, dass sie dir nicht mehr so helfen können, wie sie möchten. "Die MitarbeiterInnen setzen sich für die jungen Erwerbslosen ein, sie geben, was sie zur Verteilung zur Verfügung haben und man kann durch beständigen Kontakt und freundschaftliche Ansprache auf sie einwirken. Anstelle staatlicher Grundrechte wirkt hier ein System generalisierter Reziprozität, das der familiären Sphäre entstammt - dem allgegenwärtigen Garant ökonomischer Absicherung.

In der beschriebenen Abwesenheit und Unlesbarkeit wohlfahrtsstaatlicher Instrumente scheint die Erwerbsarbeitskrise auch zu einer Krise der Demokratie zu 
werden. Zwar sind sich junge SpanierInnen im Vergleich zu ihren deutschen Gegenübern der gesellschaftlichen Hervorbringung der Arbeitslosigkeit viel sicherer - darauf weist ihre beständige Rede vom Einsetzen der Krise und die beständige Bezugnahme auf das Kollektiv, dem „Wir“ der jungen Erwerbslosen. Einwirkungen, auch kollektive, zur Verbesserung der Bewältigungslage junger Erwerbsloser, erscheinen dennoch kaum möglich. Vor allem für diejenigen, die wie Murad aus einem Milieu mit niedrigen Bildungsabschlüssen und geringem Einkommen stammen, scheint die Lebenslage allem Einfluss entzogen zu sein. Ihre fatalistische Rede vom Glück verdeckt den gesellschaftlichen Ursprung der fehlenden Absicherung. Diejenigen, denen als Angehörige der Mittelschicht vor der Krise Absicherung und Anerkennung durch den Arbeitsmarkt sicher verfügbar schienen, suchen dagegen zunächst auch in den gesellschaftlichen Institutionen eine Lösung. Verde z. B. setzt sich politisch und kollektiv zu Wehr. Bei einer studentischen Demonstration kommt es zur Eskalation: „, Und als wir dann zurück ins Zentrum gegangen sind, ging's wieder los, mussten wir wieder rennen, weil jedes Mal, wenn einer geschrien hat, ist die Polizei wieder gekommen und hat auf den Boden geschossen oder so was. Dieser Tag war traumatisch, weißt du? [...] Ich bin dann nach Holland gegangen und hab da abgeschaltet von dieser ganzen, dieser ganzen Situation hier und den Demos und bin zurück gekommen und hab eine Beziehung angefangen und, es war so ein bisschen, ich hab das Interesse verloren in die Politik und zu den Demos zu gehen, und ich glaub, ich bin nie wieder auf eine gegangen. "Verdes Protest mündet in einer Ohnmachtserfahrung und macht dem Rückzug Platz. Fatalistischer Glaube wie bei Murad und Rückzug ins Private wie bei Verde beschreiben zwei Pole, in der sich die Unzulänglichkeit der demokratischen Auseinandersetzung im heutigen Spanien für junge Erwerbslose zeigt.

\subsubsection{Deutschland}

Ganz anders stellt sich Erwerbslosigkeit in Deutschland dar. In den Erzählungen der jungen Menschen wird deutlich, dass sie kaum noch als gesellschaftliches Phänomen verfügbar ist. Stattdessen wirkt eine rigorose Subjektivierung, die die Verantwortung für Arbeitslosigkeit bei den Einzelnen sieht und die beständig in den Interviews reproduziert wird. Obwohl Caro einige Aspekte benennen kann, die auf strukturell-institutionelle Probleme verweisen, verbleibt die Verantwortung für die Erwerbslosigkeit letztlich bei ihr: „Es ist schon sehr dieses, wenn ich das jetzt Versagen nenne, dann wird es so real, aber ja, dieses keinen Job zu finden. Und diese Frage warum. Ehm, die ich nicht beantworten kann, die aber immer da ist und die Zweifel erhöht. Also, ne, bin ich nicht genug, reicht das nicht aus, was ich gemacht hab? Reicht es nicht aus, das, was ich kann. Was mach ich falsch, weil irgendwas muss ich ja falsch machen, weil es gibt ja Jobs. " In der Folge wird Caro zur Selbstoptimiererin, die ihre vermeintlichen biografischen Makel wettzumachen sucht. Die Wahl ihrer Bewältigungsweise ,Ausbildung“ hängt eng mit der rigorosen Subjektivierung der Arbeitslosigkeit im deutschen Regime zusammen. In der Übersicht der Interviews ist es ganz besonders die Mittelklasse, die an der Subjektivierungsvorstellung leidet. Der Glaube an die Eigenverantwortung, durch die sich die Mittelschicht auszuzeichnen versucht, wird zum Bumerang. Wem Arbeitsmarkteingliederung in 
dieser gesellschaftlichen Position nicht gelingt, dessen Anerkennung ist umso mehr in Frage gestellt.

Die Institutionen des Wohlfahrtsstaates leisten der Mittelschicht kaum als passend erlebte Unterstützung. Durch ihre starke zuweisende Funktion in niedrige Beschäftigungssegmente wird der eigene Status gefährdet. Erwerbslose aus höheren Bildungs- und Einkommensschichten entziehen sich deswegen den sozialstaatlichen Instrumenten. Noch einmal Caro: „Ich mein, ich bin nicht offiziell arbeitslos gemeldet. Ehm, weil ich ganz viel Angst vor diesem habe, von wenn ich Gelder beziehe, muss ich die Jobs annehmen, die mir gegeben werden. Und da jemals wieder raus zu kommen, ist das, wo's hakt. Weil das immer wieder dann zu dem Punkt geht, dass ich was anderes machen will und dann lande ich wieder, gefühlt da, wo ich jetzt bin. Ehm, ja, mit anderer Arbeitserfahrung, aber mit keiner relevanten Arbeitserfahrung. " Für Caro können die zuweisenden Institutionen Arbeitsagentur und Jobcenter keine ,relevante Arbeitserfahrung“ vermitteln, für sie würde die Zuweisung Statusverlust mit sich bringen.

Andere Schichten sind dieser Zuweisung direkt ausgesetzt. Wer finanzielle Unterstützung benötigt, muss sich den Vorgaben der Arbeitsverwaltung fügen. Dabei spielen insbesondere die arbeitsmarktpolitischen Programme für junge Erwachsene eine Rolle. Wenn mit ihnen kein formales Bildungszertifikat, keine Orientierung auf dem Arbeitsmarkt und keine Lösung lebensweltbezogener Probleme verbunden sind, werden sie als Teil der arbeitsmarktpolitischen Bewährungsproben verstanden und zu ,irgendwelchen Maßnahmen“, die Bewältigungshandeln nicht stärken. Sergej: „Also muss ich Maßnahme machen und dann hab ich die Papieren gezeigt und bin ich gar nich hingegangen. Ich bin gar nicht einfach zur Maßnahme hingegangen. Weil ich brauchte das gar nicht. Also ich fand das irgendwie sinnlos und, ja das war auch sinnlos. " Institutionell zeigt sich der zuweisende Charakter der wohlfahrtsstaatlichen Institutionen unter anderem auch in der manageriellen Unterteilung verschiedener Hilfegruppen in der Arbeitsagentur, der Ausrichtung auf bestimmte Marktsegmente oder der Sanktionierungspraxis der Jobcenter. Fast alle InterviewpartnerInnen beziehen sich bei ihren Entscheidungen auf diese Sanktionierungspraxis. Sergej zum Beispiel berichtet von einer Sanktionierung in Zusammenhang mit der Kündigung einer Fabrikarbeit, weil er einen Schulabschluss nachholen will: „Hab ich mich einfach gekündigt. Habe ich Sperre gekriegt von drei Monaten, habe ich kein Geld gehabt, und ja. Habe ich so immer, ja okay, da waren die Freunde da, also nur die drei. Also bin ich einfach hingefahren, also ich hab da gewohnt, war ich bei dem dann. Okay, hab ich, der hat gesagt, ich brauch kein Geld von dir." Auch wenn der deutsche Wohlfahrtstaat junge Erwachsene mit eigenen sozialen Rechten versieht, sind sie nicht immer garantiert. Sergej muss auf die gemeinschaftliche Sphäre zurückgreifen und kann vorübergehend bei einem Freund wohnen. Gerade an biografischen Wendepunkten können die Institutionen Befreiungen aus gewaltvollen Verhältnissen oder wie im Fall von Sergej Ablösungen aus delinquenten Milieus gefährden. Die Konditionierung sozialer Rechte wird dann zu einer erheblichen Limitation personalen Bewältigungshandelns. Die Ergebnisse schließen hier an den häufig konstatierten konservativen und paternalistischen Charakter des deutschen Wohlfahrtsregimes an. Allerdings erscheint er in Bezug auf junge Erwerbslose noch 
verschärft, weil die Institutionen soziale Ungleichheit nicht nur passiv reproduzieren, sondern aktiv Zuweisungen vornehmen.

Wer welche Handlungsfähigkeit in den beiden Regimen ausprägen kann, ist also in hohem Maße von den ökonomischen Ressourcen anhängig, die zur Verfügung stehen. Jungen Erwerbslosen, die materiell abgesichert sind, stehen andere Optionen offen, als denen, die zunächst das materielle Überleben sichern müssen. Wer sich in einer solchen Situation befindet, der oder die etabliert Handlungsweisen, die es erlauben, das physische Überleben zu garantieren, wie es in der Bewältigungsweise „Straße“ gewährleistet ist. Junge Erwerbslose aus Schichten, die über größere Haushaltseinkommen und ökonomische Sicherheit verfügen, etablieren dagegen Handlungsweisen, die den Anerkennungsordnungen ihrer Herkunftsmilieus entsprechen, wie es in der ,Ausbildung“ erkennbar wird. Die sozialstaatlichen Institutionen stützen die ungleiche Herstellung von Handlungsfähigkeit auf unterschiedliche Weise. In Spanien werden junge Erwerbslose aus den unteren Schichten durch das Fehlen einer Basissicherung in ihrer Position gehalten. Der Teufelskreis aus notwendiger materieller Sicherung und verwehrter Verbesserung der Arbeitsmarktposition hält sie in ihrer benachteiligten gesellschaftlichen Position. In Deutschland erfolgt eine ähnliche Dynamik durch die Zuweisungen der Arbeitsverwaltung in niedrigere Arbeitsmarktsegmente, die mit dem Erhalt der Grundsicherung verbunden sind und der sich die oberen Schichten entziehen können. Die sozialstaatlichen Institutionen wählen damit in beiden Staaten einen Weg, der ungleiche Formen der Handlungsfähigkeit stützt und tragen damit zu Prozessen sozialer Schließung bei.

\section{Konsequenzen}

Für Wissenschaft und Praxis der Sozialen Arbeit erweist sich die Perspektive einer akteur*innenbasierten Sicht auf Wohlfahrtsproduktion als gewinnbringende, weil umfassende und nicht sozialpolitisch-programmatische Perspektive. Studien, die technokratisch alleine die Integration in den Arbeitsmarkt als Merkmal für Erfolg betrachten, machen nicht sichtbar, welche Leistungen junge Erwachsene erbringen, um Handlungsfähigkeit in der Situation der Erwerbslosigkeit herzustellen. Eine akteur*innenzentrierte Forschung kann dagegen umfassend die verschiedenen Anerkennungs- und ökonomischen Ordnungen freilegen, die die Lebenswelten der jungen Erwerbslosen prägen und die sie selber mit produzieren. In den identifizierten Bewältigungsweisen, die hier exemplarisch an den Beispielen „Straße“ und „Ausbildung“ erläutert wurden, gelten Regeln, Versprechungen und Limitationen, die jeweils eigene Ermöglichungen und Verwehrungen beinhalten. Mit Handlungsfähigkeit als Maßstab kann einer einseitigen Workfare-Logik widersprochen werden. So kann die Verweigerung eigenständiger sozialer Rechte für junge Erwachsene, sei es durch die Versagung einer eigenständigen Grundsicherung in Spanien oder durch Totalsanktionierungen in Deutschland zu dem Zwang führen, sich in gewaltvolle gemeinschaftliche Ordnungen einfügen zu müssen. Gleichzeitig verstärkt die Verwiesenheit auf die natürlichen Gemeinschaften soziale Ungleichheit, weil Familien und Cliquen in sehr unterschiedlichem Umfang über Ressourcen verfügen. Eine Politik der Lebensbewältigung, wie sie Lothar Böhnisch und Wolfgang Schröer 
(2013) fordern und wie sie im Bereich der Jugendberufshilfe schon lange unter dem Stichwort der lebensweltorientierten Wende diskutiert wird (z. B. Burghard und Enggruber 2010), muss Wirkungen über die Arbeitsmarktintegration hinaus betrachten, wenn sie an der Erweiterung der Handlungsspielräume junger Erwerbsloser interessiert ist.

Gleichzeitig öffnet die sozialpolitisch tradierte Regimeforschung den Blick der Sozialen Arbeit für ihre strukturelle Eingebundenheit. Sie sensibilisiert für die materiellen und symbolischen Ordnungen, in denen sowohl die Adressat*innen handeln, als auch die eigene professionelle Praxis ausgebildet wird. Vor dem Hintergrund der Regime kann sie sich die Frage stellen, wo ihr eigener Auftrag im Widerspruch zu strukturell-institutionellen Merkmalen steht und welche Rolle ihr bei der Konstitution der materiellen und symbolischen Ordnung zukommt. In Spanien zum Beispiel, wenn die individualisierenden Programme nur das Losglück erhöhen helfen oder der sozialpolitische Auftrag durch die Logiken familiärer Reziprozität überlagert wird. In Deutschland, wenn die rigorose Subjektivierung auch in der Jugendberufshilfe vorangetrieben wird oder Soziale Arbeit als Element des zuweisenden Sozialstaats Teilhabeansprüche durch Vermittlungen in prekäre Beschäftigungsverhältnisse absenkt.

Während der Vergleich der wohlfahrtskapitalistischen Regime eine produktive Folie gerade für die Reflexion in der Sozialer Arbeit darstellen kann, soll noch auf zwei Herausforderungen hingewiesen werden, die mit der Fokussierung auf Nationalstaaten einher gehen. In den Ergebnissen der empirischen Studie wird deutlich, dass sich die Möglichkeit der Etablierung von Handlungsfähigkeit junger Erwerbsloser, deren soziale Position sich innerhalb von Spanien und Deutschland ähnelt, viel stärker gleichen als der generalisierende Vergleich zwischen Ländern impliziert. So sind junge Erwachsene ohne ökonomische Absicherung in beiden Ländern viel wahrscheinlicher der Ordnung der „Straße“ überlassen als junge Erwachsene, die aufgrund der familiären oder gesellschaftlich zur Verfügung gestellten Absicherung befähigt werden und verpflichtet sind, durch weitere Ausbildung ihre Position am Markt zur verbessern. Die Rede von dem einen wohlfahrtskapitalistischen Regime läuft Gefahr zu verschleiern, dass die damit implizierten Ordnungen je nach sozialer Lage sehr unterschiedlich ausgestaltet sein können. Dabei sind es grade die Regime, die für Menschen in unterschiedlichen gesellschaftlichen Positionen unterschiedliche Handlungsspielräume bereithalten. Die wohlfahrtsstaatlichen Regime haben einen Einfluss darauf, wer in welche Position gerät - etwa, wenn sich junge Erwerbslose in Spanien aufgrund der fehlenden Absicherung in die verpflichtenden reziproken Gemeinschaften begeben und in Deutschland die gesellschaftliche Zuweisung akzeptieren müssen, wenn sie wohlfahrtsstaatliche Absicherungen in Anspruch nehmen.

Zum anderen kann der Vergleich der Regime den Blick auf inter- und supranationale Zusammenhänge verstellen, wie sich besonders bei der Betrachtung von Deutschland und Spanien zeigt. Der gemeinsame europäische Binnenmarkt und die gemeinsame Währung ist für den Bauboom in Spanien ebenso verantwortlich wie für das Platzen der Immobilienblase und die daraus folgende Beschäftigungskrise seit 2008. Der Europäisierung des Marktes steht jedoch keine gleichwertige europaweite, soziale Absicherung gegenüber. Eine dem Kapitalismus eingeschriebene 
Idee des Sozialen, wie sie bei Karl Polanyi als Doppelbewegung (1978 [1944]) oder bei Eduard Heimann (1980 [1929]) als konservativ-revolutionäres Doppelwesen konzipiert ist, ist auf der Ebene der europäischen Institutionen nicht erkennbar. Sozialpolitik gestaltet sich stattdessen in Abhängigkeit einer Logik, die bestimmt ist durch Austerität, dem Festhalten am Euro als gemeinsamer Währung und der NichtVergemeinschaftung anderer Bereiche der Wirtschafts- und Sozialpolitik. Die so genannte Jugendgarantie bietet weder eine Ausstattung mit sozialen Rechten noch ein Antwort auf die Probleme am Arbeitsmarkt. Politik diskreditiert sich hier selbst, weil ein soziales Recht trotz der Verwendung des Wortes „Garantie“ nicht gewährleistet wird.

In der Folge polarisiert sich die Lebenslage der europäischen Bevölkerung und die soziale Spaltung vollzieht sich nicht nur zwischen Staaten, sondern auch zwischen Schichten und Generationen (Offe 2016). In den Lebenswelten der jungen Menschen zeigen sich die mangelnde Integrationskraft des Marktes und der fehlende sozialpolitische Ausgleich auch als ein Demokratieproblem. Wie am Beispiel von Murad und Verde deutlich wird, leben junge Erwerbslose in Spanien in ihrer eigenen Wahrnehmung schon heute in einer direkt erfahrbaren ,wirtschaftlich entmachteten Postdemokratie" (Streeck 2015, S. 262), während in Deutschland auch mit Hilfe der Sozialen Arbeit die rigorose Subjektivierung die gesellschaftlichen Ursachen von Arbeitslosigkeit verschüttet. Die Verbindung von Regime- und Akteur*innenforschung zeigt, dass in dieser Situation einseitiger Marktpolitik junge Menschen aufgefordert sind, Anerkennung und materielle Absicherung in Ordnungen herzustellen, die auf Loyalitäten und reziproken Verpflichtungen statt auf sozialen Rechten beruhen. Die Verbindung von Regime- und Akteur*innenperspektive kann dazu beitragen, die komplexen Wechselwirkungen zu verstehen, die nicht auf die Institutionen von Markt und Staat beschränkt bleiben und freilegen, in welchen eigenen Ordnungen Menschen verschiedener Lebenslagen ihr Streben nach Handlungsfähigkeit verwirklichen.

Open Access Dieser Artikel wird unter der Creative Commons Namensnennung 4.0 International Lizenz (http://creativecommons.org/licenses/by/4.0/deed.de) veröffentlicht, welche die Nutzung, Vervielfältigung, Bearbeitung, Verbreitung und Wiedergabe in jeglichem Medium und Format erlaubt, sofern Sie den/die ursprünglichen Autor(en) und die Quelle ordnungsgemäß nennen, einen Link zur Creative Commons Lizenz beifügen und angeben, ob Änderungen vorgenommen wurden.

\section{Literatur}

Achatz, J., Fehr, S., Schels, B., \& Wolff, J. (2012). Wovon junge Arbeitslose im SGB II am meisten profitieren. IAB Kurzbericht, 2012(6), 1-8.

Baethge, M. (2014). Das deutsche Qualifizierungsmodell - Blaupause für Europa im Kampf gegen Jugendarbeitslosigkeit? In K. Dörre, K. Jürgens \& I. Matuschek (Hrsg.), Arbeit in Europa (S. 107-124). Frankfurt am Main: Campus.

Banyuls, J., \& Recio, A. (2012). Spain: The nightmare of Mediterranean neoliberalism. In S. Lehndorf (Hrsg.), A triumph of failed ideas. European models of capitalism in the crisis (S. 199-218). Brussels: Etui.

Bareis, E. (2012). Nutzbarmachung und ihre Grenzen - (Nicht-)Nutzungsforschung im Kontext von sozialer Ausschließung und der Arbeit an der Partizipation. In E. Schimpf \& J. Stehr (Hrsg.), Kritisches Forschen in der Sozialen Arbeit (S. 291-314). Wiesbaden: VS.

Böhnisch, L., Lenz, K., \& Schröer, W. (2009). Sozialisation und Bewältigung. Eine Einführung in die Sozialisationstheorie der Moderne. Weinheim: Juventa. 
Böhnisch, L., \& Schröer, W. (2001). Pädagogik und Arbeitsgesellschaft. Historische Grundlagen und theoretische Ansätze für eine sozialpolitisch reflexive Pädagogik. Weinheim: Juventa.

Böhnisch, L., \& Schröer, W. (2013). Soziale Arbeit - eine problemorientierte Einführung. Bad Heilbrunn: Julius Klinkhardt.

Bourdieu, P. (2000). Die zwei Gesichter der Arbeit. Interdependenzen von Zeit- und Wirtschaftsstrukturen am Beispiel einer Ethnologie der algerischen Übergangsgesellschaft. Konstanz: UVK.

Bundesagentur für Arbeit (BA) (2016). Arbeitsmarkt 2015. Nürnberg: Bundesagentur für Arbeit.

Bundesministerium für Bildung und Forschung (BMBF) (2016). Berufsbildungsbericht 2016. Bonn: BMBF.

Burghardt, H., \& Enggruber, R. (2010). Soziale Dienstleistungen am Arbeitsmarkt in professioneller Reflexion Sozialer Arbeit (S. 13-59). Berlin: Frank \& Thimme.

Cabasés Piqué, À., \& Pardell Veà, A. (2014). Una visión crítica del Plan de Implantación de la Garantía Juvenil en España. Albacete: Bomarzo.

Chevalier, T. (2016). Varieties of youth welfare citizenship: towards a two dimension typology. Journal of European Social Policy, 26(2016), 3-19.

Dörre, K., Scherschel, K., Booth, M., Haubner, T., Marquardsen, K., \& Schierhorn, K. (2013). Bewährungsproben für die Unterschicht? Soziale Folgen aktivierender Arbeitsmarktpolitik. Frankfurt am Main: Campus.

Esping-Andersen, G. (1990). The three worlds of welfare capitalism. Cambridge: Polity Press.

Eurostat (2015). European Union Labour Force Survey. http://appsso.eurostat.ec.europa.eu/nui/show.do? dataset=lfsa_urgaed\&lang=en. Zugegriffen: 06. Jan. 2017.

Eversberg, D. (2014). Dividuell aktiviert. Wie Arbeitsmarkt Subjektivitäten produziert. Frankfurt am Main: Campus.

Flick, U. (2011). Qualitative Sozialforschung. Eine Einführung. Reinbek: Rowohlt.

Gobierno de España (2013a). Estrategia de Emprendimiento y Empleo Joven 2013/2016 (EEEJ). http:// www.empleo.gob.es/ficheros/garantiajuvenil/documentos/EEEJ_Documento.pdf. Zugegriffen: 04. Januar 2017.

Gobierno de España (2013b). Plan Nacional de Implantación de la Garantía Juvenil en España. http:// www.empleo.gob.es/es/estrategia-empleo-joven/destacados/plannacionalgarantiajuvenil_es.pdf. Zugegriffen: 04. Januar 2017.

Götz, S., Ludwig-Mayerhofer, W., \& Schreyer, F. (2010). Sanktionen im SGB II. IAB Kurzbericht, 2010(10), 1-8.

Graßhoff, G. (2013). Adressaten, Nutzer, Agency. Akteursbezogene Forschungsperspektiven in der Sozialen Arbeit. Wiesbaden: Springer VS.

Gutiérrez, E. (2014). Nova pobresa i renda mínima d'inserció. Dossier Catalunya Social, Propostes des del Tercer Sector. Taula d'entitats del Tercer Sector Social de Catalunya. http://www.tercersector.cat/ sites/default/files/dossier_nova_pobresa_i_renda_minima_dinsercio_maig_2014_0.pdf. Zugegriffen 06. Jan. 2017.

Heimann, E. (1980). Soziale Theorie des Kapitalismus. Theorie der Sozialpolitik. Frankfurt am Main: Suhrkamp.

Herzog, K. (2015). Schulden und Alltag. Arbeit mit schwierigen finanziellen Situationen und die (Nicht-)Nutzung von Schuldnerberatung. Münster: Westfälisches Dampfboot.

Institut für Arbeitsmarkt- und Berufsforschung (2016). IAB Prognose 2016. IAB Kurzbericht, 2016(6), $1-12$.

Kessl, F. (2013). Soziale Arbeit in der Transformation des Sozialen. Eine Ortsbestimmung. Wiesbaden: SpingerVS.

Kolbe, C. (2013). Arbeitsmarktpolitik: Übergänge in Arbeit. In W. Schröer, B. Stauber, A. Walther, L. Böhnisch \& K. Lenz (Hrsg.), Handbuch Übergänge (S. 1091-1114). Weinheim: Juventa.

Lehndorff, S. (2014). Die Agenda-Legende: Die neue Karriere des „Modells Deutschland“ in Europa. In K. Dörre, K. Jürgens \& I. Matuschek (Hrsg.), Arbeit in Europa. Marktfundamentalismus als Zerreissprobe (S. 165-179). Frankfurt am Main: Campus.

Lessenich, S. (2008). Die Neuerfindung des Sozialen. Der Sozialstaat im flexiblen Kapitalismus. Bielefeld: transcript.

Lessenich, S., \& Ostner, I. (1998). Welten des Wohlfahrtskapitalismus - Wandel der Wohlfahrtsstaatenforschung. Der Sozialstaat in vergleichender Perspektive. Frankfurt am Main: Campus.

Lindner, F. (2013). Banken treiben Eurokrise. IMK Report, Nr. 82, Juni 2013.

López, I., Rodriguez, E. (2012). Das spanische Modell, Prokla 166, 42(1), 113-134.

Ludwig-Mayerhofer, W., Behrend, O., \& Sondermann, A. (2009). Auf der Suche nach der verlorenen Arbeit. Arbeitslose und Arbeitsvermittler im neuen Arbeitsmarktregime. Konstanz: UVK. 
Merton, R. K. (2016). Sozialstruktur und Anomie. In D. Klimke \& A. Lignaro (Hrsg.), Kriminologische Grundlagentexte (S. 245-268). Wiesbaden: Springer VS.

Mohr, K. (2009). Von „Welfare to Workfare“? Der radikale Wandel der deutschen Arbeitsmarktpolitik. In S. Bothfeld, W. Sesselmeier \& C. Bogedan (Hrsg.), Arbeitsmarktpolitik in der sozialen Marktwirtschaft (S. 49-60). Wiesbaden: SpringerVS.

Ministerio de Sanidad, Servicios Sociales, e Igualdad (MSSSI) (2015). El servicio público de servicios sociales. Informe de rentas mínimas de inserción. Año 2014. https://www.msssi.gob.es//ssi/ familiasInfancia/inclusionSocial/serviciosSociales/RentasMinimas/Docs/InformeRMI2014.pdf. Zugegriffen: 04. Januar 2017.

Offe, C. (2016). Europa in der Falle. Berlin: Suhrkamp.

Olk, T. (2008). Soziale Arbeit und Sozialpolitik. Notizen zu einem ambivalenten Verhältnis. In Bielefelder Arbeitsgruppe 8 (Hrsg.), Soziale Arbeit in Gesellschaft (S. 287-298). Wiesbaden: Springer VS.

Polany, K. (1978). The Great Transformation. Politische und ökonomische Ursprünge von Gesellschaften und Wirtschaftssystemen. Frankfurt am Main: Suhrkamp.

Raitelhuber, E. (2013). Agency und Übergänge. In W. Schröer, B. Stauber, A. Walther, L. Böhnisch \& K. Lenz (Hrsg.), Handbuch Übergänge (S. 99-140). Weinheim: Juventa.

Reißig, B. (2016). Übergänge von der Schule in den Beruf. Forschungsbefunde und Herausforderungen. In W. Kühnel \& D. Zifonun (Hrsg.), Übergangspraxis. Zur Gestaltung des Übergangs von der Schule in den Beruf (S. 12-28). Weinheim: Juventa.

van Rießen, A. (2016). Zum Nutzen Sozialer Arbeit. Theaterpädagogische Maßnahmen im Übergang zwischen Schule und Erwerbsarbeit. Wiesbaden: Springer.

Rosenthal, G., Köttig, M., Witte, N., \& Blezinger, A. (2006). Biografisch-narrative Gespräche mit Jugendlichen. Chancen für das Selbst- und Fremdverstehen. Opladen: Barbara Budrich.

Scherr, A. (2012). Soziale Bedingungen von Agency. Soziologische Eingrenzung einer sozialtheoretisch nicht auflösbaren Paradoxie. In S. Bethmann, C. Helfferich, H. Hoffmann \& D. Niermann (Hrsg.), Agency. Qualitative Rekonstruktionen und gesellschaftstheoretische Bezüge von Handlungsmächtigkeit (S. 99-121). Weinheim: Juventa.

Seco, A.J., \& Gonzales, G. B. (2000). La asistencia social y los servicios sociales en España. Madrid.: Boletín oficial del estado.

Serrano, P. A. (2007). Activation Regimes in Europe: A Clustering Exercise. In P. A. Serrano \& L. Magnusson (Hrsg.), Reshaping Welfare States and Activation Regimes in Europe (S. 275-316). Brüssel: Peter Lange.

Servicio Público de Empleo Estatal (SEPE) (2015). Prestaciones por Desempleo. http://www.empleo.gob. es/es/estadisticas/anuarios/2015/PRD/PRD.pdf. Zugegriffen: 06. Jan. 2017.

Stauber, B, Pohl, A. \& Walther, A. (Hrsg.) (2007). Subjektorientierte Übergangsforschung. Rekonstruktion und Unterstützung biografischer Übergänge junger Erwachsener. Weinheim und München: Juventa.

Steinert, H., \& Pilgram, A. (2001). Welfare policy from below. Struggles against social exclusion in Europe. Burlington: Ashgate.

Strauss, A. (1998). Grundlagen qualitativer Sozialforschung. München: Wilhelm Fink.

Strauss, A., \& Corbin, J. (1994). Grounded theory methodology - an overview. In N. Denzin \& Y. Lincoln (Hrsg.), The Sage handbook of qualititative research (S. 273-285). Thousand Oaks: Sage.

Streeck, W. (2015). Gekaufte Zeit. Die vertagte Krise des demokratischen Kapitalismus. Berlin: Suhrkamp.

Walther, A. (2011). Regimes der Unterstützung im Lebenslauf. Ein Beitrag zum internationalen Vergleich in der Sozialpädagogik. Opladen: Barbara Budrich.

Walther, A. (2013). International vergleichende Übergangsforschung. In W. Schröer, B. Stauber, A. Walther, L. Böhnisch \& K. Lenz (Hrsg.), Handbuch Übergänge (S. 1091-1114). Weinheim: Juventa.

Walther, A., Walter, S., \& Pohl, A. (2007). „Du wirst echt in eine Schublade gesteckt...“. Junge Frauen und Männer im Übergangssystem zwischen Wahlbiographie und Cooling-Out. In B. Stauber, A. Pohl \& A. Walther (Hrsg.), Subjektorientierte Übergangsforschung. Rekonstruktion und Unterstütrzung biografischer Übergänge junger Erwachsener (S. 97-128). Weinheim: Juventa.

Weber, E. (2014). Das Ziel der Vollbeschäftigung in Deutschland. Fern, aber erreichbar. IAB Kurzbericht, 2014(15), 1-11.

Weber, J. (2014). Social integration through employment - Desintegration through employment? Dynamics of activation and precarity in school-to-work transitions in Germany. In: The International Academic Forum (IAFOR), The Asian Conference on the Social Sciences. Conference Proceedings 2014. (S. 745-760). http://iafor.org/archives/proceedings/ACSS/ACSS2014_proceedings.pdf. Zugegriffen: 04. Januar 2017.

Winkler, G., \& Degele, N. (2009). Intersektionalität. Zur Analyse sozialer Ungleichheiten. Bielefeld: transcript. 